\title{
UN NUEVO ENFOQUE PARA LA GESTIÓN DE LAS POLÍTICAS DE INNOVACIÓN, EN FUNCIÓN DEL DESARROLLO SOSTENIBLE
}

\author{
Milagros Acacia Saucedo Nardo \\ Dra. en Ciencias filosófica GECYT \\ Empresa de gestión del conocimiento y la tecnología \\ milagros@gecyt.cu
}

\section{RESUMEN}

La oleada globalizadora que caracteriza al siglo XXI se distingue de las anteriores por la velocidad con que ocurren los cambios tecnológicos y su impacto en las políticas y en los modelos organizacionales de gestión de la innovación. La marcha de los acontecimientos sitúa ante nosotros, cada vez más insistentemente, problemas relativos a la ciencia, tales como su apropiado uso en la sociedad, la militarización de la ciencia, las relaciones de esta con los gobiernos, el secreto científico, la libertad de la ciencia, el lugar de la misma en la educación y en la cultura general. ¿Cómo resolver tales problemas? Precisamente desde la perspectiva de la integración ciencia, innovación y sociedad se continúa el reto de nuevos estudios multidisciplinarios para profundizar teórica y prácticamente, en cómo lograr prospectivamente sistemas de innovación más competitivos en una sociedad más humanizada. Ante el reto de una nueva visión en el siglo XXI, la autora conceptualiza, propone, participa en la dirección y sistematiza una experiencia novedosa para la gestión del conocimiento, e incorpora un nuevo enfoque para la reflexión socio-filosófica en el debate actual, sobre la construcción de nuevos paradigmas para la gestión de la innovación en la época actual. El valor científico del trabajo se resume en su novedad, al no existir experiencia anterior en Cuba, de estudios socio-filosóficos sobre la temática en particular, la autora aporta un nuevo enfoque para la gestión de las políticas de innovación integrado coherentemente con el medio ambiente, como base conceptual aplicado a un estudio de caso en la provincia de Villa Clara, Cuba, de un nuevo modelo alternativo de sistema de innovación más competitivo e integrado, para propiciar el avance sostenible de la sociedad: MOGECITECMA.

Palabras clave: Innovación; Sistemas de innovación; Innovación sostenible. 


\section{INTRODUCCIÓN}

La oleada globalizadora que caracteriza al siglo XXI se distingue de las anteriores por la velocidad con que ocurren los cambios tecnológicos y su impacto en los modelos organizacionales de gestión de la ciencia y la innovación.

Los estudios sobre ciencia, tecnología y sociedad, constituyen un importante nicho de investigación donde se trata de entender el fenómeno científico-tecnológico en su relación con el contexto social. En el siglo XXI es un campo de estudio bien organizado institucionalmente en universidades, redes y centros educativos de numerosos países, sobre todo desarrollados.

El enfoque general utilizado es de carácter crítico, con respecto a la clásica visión esencialista y triunfalista de la ciencia y la tecnología, y también de carácter interdisciplinario y transdisciplinario, pues concurren en él disciplinas como la filosofía y la historia de la ciencia y la tecnología, la sociología del conocimiento científico, la teoría de la educación, la economía de lo tecnológico, las ciencias de la gestión y la dirección empresarial.

El cambio académico de la imagen de la ciencia y la tecnología es un proceso que comienza en los años 70 y que hoy se halla en fase de intenso desarrollo. Se trata de los estudios ciencia, tecnología y sociedad (CTS). La clave se encuentra en presentar la ciencia-tecnología no como un proceso o actividad autónoma que sigue una lógica interna de desarrollo en su funcionamiento óptimo, sino como un proceso o producto inherentemente social donde los elementos no técnicos (por ejemplo valores morales, convicciones religiosas, intereses profesionales, presiones económicas, etc.) desempeñan un papel decisivo en su génesis y consolidación. La complejidad de los problemas abordados y su flexibilidad interpretativa desde distintos marcos teóricos, hacen necesaria la presencia de esos elementos no técnicos bajo la forma de valores o de intereses contextuales.

A su vez, numerosos autores llaman la atención sobre las problemáticas consecuencias, de naturaleza ambiental y social, que tiene el actual y vertiginoso desarrollo científico-tecnológico, unas consecuencias sobre las que es necesario reflexionar y proponer líneas de acción. En el punto de mira de esas líneas se encontrarían problemas como el de la equidad en la distribución de costes ambientales de la innovación tecnológica (e.g. experimentación con organismos modificados genéticamente), el uso inapropiado de descubrimientos científicos (e.g. diferencias sexuales en tipos de conducta inteligente), las implicaciones éticas de algunas tecnologías (e.g. uso comercial de la información genética, madres de alquiler), la aceptación de los riesgos de otras tecnologías (e.g. energía nuclear, fertilizantes químicos), o incluso el cambio en la naturaleza del ejercicio del poder debido a la institucionalización actual del asesoramiento experto (problema de la tecnocracia). 
Así se ha conformado un nuevo escenario socioeconómico donde la competencia, los recursos intangibles, la información, la informatización, y el aprender y desaprender continúo en el capital humano, exigen entre los retos actuales, nuevas formas para la gestión del conocimiento entre los agentes de la sociedad, que les permita a través de enfoques, modelos, redes, y herramientas renovadas la gerencia más eficiente y eficaz de la ciencia en función del desarrollo social sostenible.

El tema de la ciencia y su papel para el desarrollo social ha sido y sigue siendo objeto de investigación recurrente en el pensamiento contemporáneo. Precisamente desde la perspectiva de la integración ciencia, tecnología y sociedad se continúa el reto de nuevos estudios multidisciplinarios para profundizar teórica y prácticamente, en cómo lograr una sociedad científica y tecnológica más humanizada, capaz de rebasar la crisis de los paradigmas actuantes.

Las tendencias mencionadas y la necesidad de la ciencia cubana de insertarse en el mundo actual tan complejo y cambiante, impone pensar y generar cambios culturales y organizacionales capaces de lograr la renovación de enfoques y prácticas para la gestión de la ciencia y las políticas de innovación al ritmo requerido y acorde a las peculiaridades de Cuba.

Con un enfoque socio-filosófico MOGECITECMA aporta indicadores para contribuir a responder la interrogante, ¿cómo gestionar la ciencia y la innovación en redes de gestión del conocimiento dirigidas a propiciar el desarrollo sostenible de la sociedad?

El propósito como se verá más adelante es estimular la reflexión sobre cómo lograr avanzar hacia una sociedad científica y tecnológicamente más humanizada, capaz de rebasar la crisis de los paradigmas actuantes, y propiciar un modelo referente con un enfoque hacia el desarrollo sostenible de las empresas, organizaciones y la sociedad, para la toma de decisiones de los gobiernos locales y regionales en cuanto a la gestión integrada en redes de la ciencia y la innovación y el medio ambiente.

\subsection{LA GESTION DE LA CIENCIA Y LA INNOVACIÓN. ANTECEDENTES Y PROBLEMÁTICA EN LA SOCIEDAD ACTUAL}

Un análisis actual sobre los antecedentes de la gestión de la ciencia y la innovación confirma el papel de la ciencia como elemento dinamizador de la sociedad en todas las etapas de su desarrollo.

El concepto de Gestión proviene del latín gestio: acción administrar. Gestión, dirección. Actividad profesional tendiente a establecer los objetivos y medios de su realización, a precisar la organización de sistemas, a elaborar la estrategia del desarrollo y a ejecutar la gestión del personal. En el concepto gestión es muy importante la acción, del latín actionem; que significa toda manifestación

Revista de Administração e Inovação, São Paulo, v. 7, n. 3, p. 79-93, jul./set. 2010 
Un nuevo enfoque para la gestión de las políticas de innovación, en función del desarrollo sostenible.

de intención o expresión de interés capaz de influir en una situación dada. El énfasis que se hace en la acción, en la definición de gestión es la diferencia que se tiene con el concepto de administración.

A criterio de la autora el término gestión, designa la acción de dirección del proceso del cual forman parte las actividades cotidianas de planificar (trazar políticas y estrategias), organizar, controlar y evaluar el cumplimiento de objetivos de una organización dada, para cumplir la misión y el objeto social para lo cual fue creada.

La concepción clásica de las relaciones entre ciencia, tecnología y sociedad, todavía presente en buena medida en diversos ámbitos del mundo académico y medios de divulgación, es una concepción esencialista y triunfalista. Puede resumirse en una simple ecuación:

\section{+ ciencia $=+$ tecnología $=+$ riqueza $=+$ bienestar social}

Mediante la aplicación del método científico (como una suerte de combinación de razonamiento lógico y observación cuidadosa) y el acatamiento de un severo código de honestidad profesional, se espera que la ciencia produzca la acumulación de conocimiento objetivo acerca del mundo. Ahora bien — se nos advierte en esta visión clásica - la ciencia sólo puede contribuir al mayor bienestar social si se olvida de la sociedad para buscar exclusivamente la verdad. Análogamente, sólo es posible que la tecnología pueda actuar de cadena transmisora en la mejora social si se respeta su autonomía, si se olvida de la sociedad para atender únicamente a un criterio interno de eficacia técnica. Ciencia y tecnología son presentadas así como formas autónomas de la cultura, como actividades valorativamente neutrales, como una alianza heroica de conquista de la naturaleza (Echeverria, 1995; González García, Lopez Cerezo, \& Luján, 1996, p. 11).

El mundo se vuelve cada día más cambiante y complejo. La innovación no puede ya analizarse con un enfoque, donde predomine sólo lo tecnológico y el mercado. Necesita ser tratada y desarrollada en un contexto transdisciplinario donde los aspectos organizacionales, científicos, tecnológicos, sociales y ambientales se vean de forma dialéctica.

Para contribuir al debate del pensamiento filosófico latinoamericano sobre la relación ciencia, tecnología y sociedad la autora incorpora un nuevo enfoque del problema, y es precisamente no centrar el análisis en el condicionamiento social de la ciencia o en la explicación de la ciencia como sostén de la sociedad y/o viceversa, sino ampliar la reflexión hacia la relación dialéctica: SOCIEDADCIENCIA-SOCIEDAD SOSTENIBLE (SCSs) demandada hoy con prioridad, para la conservación presente y futura de la vida en el planeta .

Revista de Administração e Inovação, São Paulo, v. 7, n. 3, p. 79-93, jul./set. 2010 
El nuevo enfoque se explica a través del modelo propuesto en el estudio de caso e incluye en el proceso cíclico de implantación, tres fases esenciales:(S) sociedad demandante, (C) la ciencia oferente y como resultado (Ss) el avance hacia la sociedad sostenible como paradigma para las presentes y futuras generaciones. Este proceso estará condicionado por los cambios presentes y futuros en los entornos económicos y sociales de los diferentes países y por la objetiva intercomunicación, interdependencia e interpenetración en términos de la creación humana, cultura material y espiritual a nivel de todo el globo terráqueo.

\section{LA CIENCIA Y LA INNOVACIÖN TECNOLÓGICA EN CUBA. NECESIDAD Y PROPUESTA DE UN ENFOQUE DIFERENTE PARA SU PERFECCIONAMIENTO}

Con el objetivo de perfeccionar la implementación del enfoque integrado de sistema de ciencia, innovación tecnológica y medio ambiente, (en lo adelante SCIT y MA) lo que constituye una novedad a nivel nacional. Fue necesario elaborar nuevas herramientas que facilitaran la cultura imprescindible para el auto perfeccionamiento gerencial del SCIT Y MA, y lograr una mejor dinámica de entrecruzamiento en una red entre los que producen bienes materiales y los que generan conocimientos, en función de incidir más aceleradamente en el paulatino crecimiento económico social sostenible territorial.

En las interrelaciones que se producen en el marco de la red de gestión de conocimientos del SCIT y MA y (ver Figura 1) se ubica el Gobierno como elemento decisor superior del sistema, ya que a través de sus diferentes estructuras el gobierno orienta, organiza y dirige el desarrollo socioeconómico de la provincia, para lo cual tiliza las potencialidades que le brinda la ciencia y la innovación a través de los elementos integrantes del SCIT y MA. 


\section{RED PARA LA GESTION DE INNOVACION}

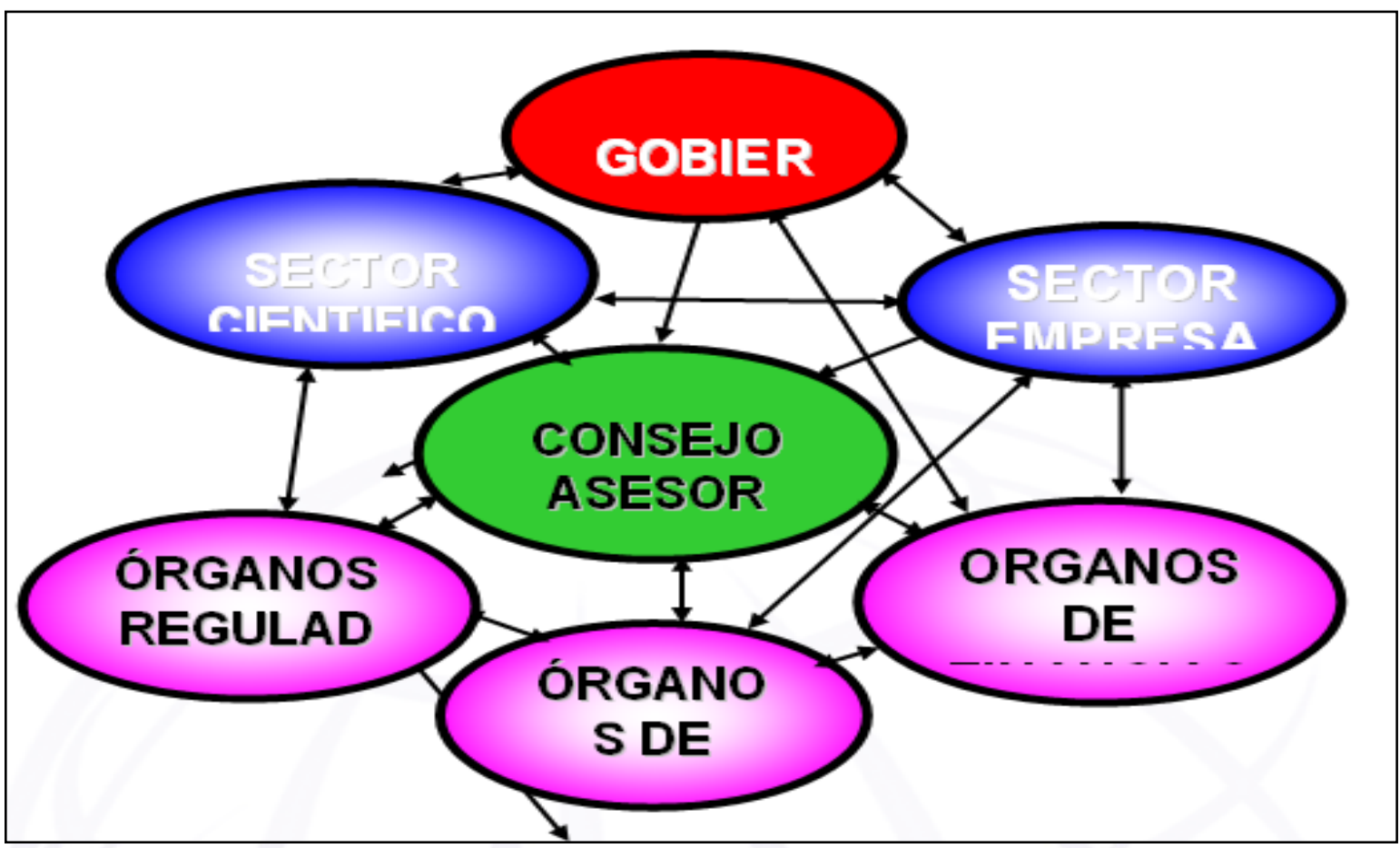

Figura 1 - Estructura para una red gestión de ciencia e innovación. Fuente: Nardo (2006).

El SCIT y MA es el conjunto de la estructura organizativa para la identificación y planificación de actividades, procesos, procedimientos y los recursos necesarios que contribuyen a la solución de problemas científicos, tecnológicos y ambientales, sobre la base de los principios del desarrollo sostenible. Funciona como una red de gestión del conocimiento y está integrado por todos los agentes y organizaciones involucrados activamente en el desarrollo de la capacidad de innovación del território (Nardo, 2006). Estos agentes establecen múltiples interrelaciones e interconexiones a través de la red en el proceso de gestión de ciencia e innovación.

\section{MOGECITECMA. SÍNTESIS DE UN MODELO ALTERNATIVO PARA LA GESTIÓN INTEGRADA DE LA CIENCIA, LA INNOVACIÓN Y EL MEDIO AMBIENTE}

Para participar en este amplio y complicado debate, la autora concibe un nuevo modelo que tiene como objetivo general: Proponer un enfoque alternativo para la gestión de redes del conocimiento y la innovación, dirigido hacia el desarrollo sostenible, que propicie una gestión en las empresas y 
organizaciones más eficiente y en correspondencia las prioridades del desarrollo territorial y de la sociedad.

\section{¿Qué es el modelo MOGECITECMA?}

MOGECITECMA, es un modelo de gestión organizacional para redes de gestión integrada de ciencia, innovación y medio ambiente, creado para las empresas y entidades que se orienten hacia la gestión integrada en redes y procesos con miras hacia el desarrollo sostenible.

Proporciona procedimientos para diagnosticar, planificar, evaluar y controlar el impacto de las entidades hacia lo interno de la organización y hacia la sociedad.

MOGECITECMA se representa gráficamente en la Figura 2, y muestra de forma simplificada, el proceso seguido para su aplicación en Villa Clara (Provincia central de Cuba) para la gestión integrada de la ciencia y la innovación, en condiciones amigables con el medio ambiente y en función del desarrollo territorial y el mejoramiento de la calidad de vida de la sociedad.

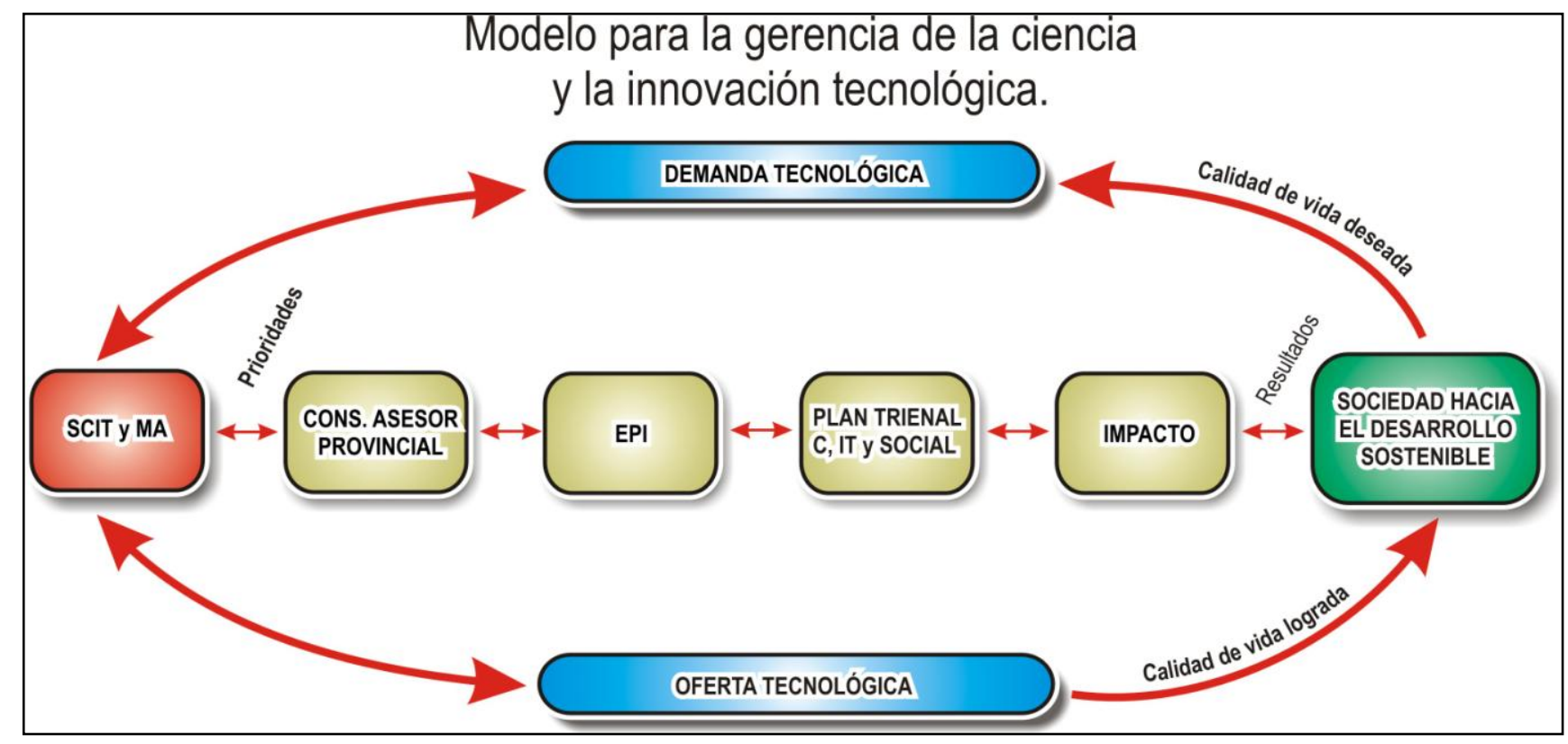

Figura 2 - Modelo para la gerencia de la ciencia y la innovacion tecnológica Fuente: Nardo (2006).

El modelo está sustentado en los siguientes principios: a) la objetiva intercomunicación, interdependencia e interpenetración para la gestión de los conocimientos y tecnologías que generen los diferentes actores de las organizaciones; b) la integración en redes, la cooperación interactiva científica, tecnológica y productiva para compartir el despliegue de tecnologías, y procedimientos que 
Un nuevo enfoque para la gestión de las políticas de innovación, en función del desarrollo sostenible.

son elementos de la competitividad en las organizaciones; c)la transdisciplinariedad y la interdisciplinariedad, para comprender y asimilar la heterogeneidad de las demandas de la sociedad y los complejos problemas del entorno y corresponder con la excelencia de los resultados, manteniendo a su vez la correspondencia con las prioridades del desarrollo territorial; d)La flexibilidad y evaluación participativa y sistemática de los resultados de su funcionamiento por la sociedad, que es su principal cliente, para mantener la innovación como base de funcionamiento e incidir decisivamente en el avance hacia el desarrollo sostenible. .

A criterio de la autora MOGECITECMA, aplica una visión renovada sobre la relación dialéctica ciencia, tecnología y sociedad, para organizar una red de gestión de conocimientos y lograr una gestión más eficiente de la ciencia integrada al medio ambiente y propiciar resultados decisivos para la calidad de vida de los hombres, a través de la identificación de las demandas de la sociedad, su ordenamiento por prioridades, y la planificación de programas territoriales y asignación de financiamiento para la ejecución de proyectos con ofertas tecnológicas e impactos tangibles en el desarrollo económico y social territorial.

El ciclo del modelo se expresa en la interacción dialéctica SOCIEDAD-CIENCIA-SOCIEDAD SOSTENIBLE (SCSs), demandada hoy con prioridad para la conservación presente y futura de la vida en el planeta. El enfoque del modelo elaborado incluye en el proceso cíclico tres fases esenciales: la sociedad demandante, la ciencia oferente y como resultado, una sociedad cualitativamente superior por el valor añadido que le incorporan los impactos de la ciencia a través del uso de tecnologías limpias para el avance hacia el desarrollo sostenible.

En MOGECITECMA, es la sociedad, y no solo el mercado o la competitividad empresarial el punto de partida y destino final del proceso de la gestión de la ciencia y la innovación tecnológica, y sin ser absolutos, es en última instancia en la sociedad como totalidad donde se expresa a través de la praxis social lo acertado o no de las acciones de la ciencia, la it y medio ambiente, la satisfacción o no de las demandas siempre crecientes de los hombres, y donde la población mejora o no su calidad de vida. El modo de producción de la vida material condiciona el proceso de la vida social, política y espiritual en general, el citado principio materialista forma parte de la base de la nueva concepción y enfoque propuesto para la gestión e implementación del modelo diseñado.

La autora concibe en la investigación el concepto de calidad de vida como: mejoras en la salud y bienestar de los hombres, seguridad de empleo, calidad ambiental, democracia, cultura, ingresos económicos, estimulo a la iniciativa y el aprendizaje, humanismo, justicia, seguridad social y la igualdad de oportunidades para el desarrollo de todos los miembros de la sociedad.

Revista de Administração e Inovação, São Paulo, v. 7, n. 3, p. 79-93, jul./set. 2010 
¿Qué premisas básicas o elementos esenciales condicionan la aplicación de MOGECITECMA en una entidad?

- A. Existencia de una organización o estructura, que constituya el soporte para la gestión del conocimiento y la innovación, integrada con el medio ambiente, que logre la transformación gradual y cualitativa en las organizaciones, directivos, técnicos y en los trabajadores del concepto y cultura del plan de la economía y plan de ciencia y técnica por el concepto del plan de ciencia, innovación y sostenibilidad económica y ambiental.

- B. Concepción e implementación en la empresa o entidad de una política y estrategia, integrada de ciencia, innovación y medio ambiente.

- C. Aplicación sistemática de indicadores y requisitos, para la evaluación del impacto del conocimiento y la innovación en la eficiencia empresarial en función de su contribución armónica con las prioridades de estrategia territorial y el avance hacia la sostenibilidad ambiental.

Las herramientas básicas para la aplicación del modelo son:

1. Sistema de Gestión de la innovación a través de la ejecución de proyectos, acciones de innovación, de desarrollo tecnológico y del financiamiento aprobado en la entidad.

2. Sistema de Gestión Ambiental, a partir del diagnóstico ambiental y la implementación del plan de acciones para avanzar en la eliminación de los riesgos ambientales y lograr la disminución de impactos negativos hacia el ecosistema.

3. Sistema de Propiedad Industrial, para el diseño e implementación del sistema en la entidad para la protección legal de los intangibles de la organización.

4. Sistema de Gestión de la Calidad, para la acreditación, certificación y aplicación de las normas de calidad vigentes. Seguridad y salud en el trabajo. 
5. Sistemas de Gestión para atención al capital humano, la comunicación empresarial, la información, infraestructura y sistema de información e informatización y su uso para la toma de decisiones.

6. Sistema de mercadotecnia y control interno, con la identificación de financiamiento anual aprobado y ejecutado para acciones y/o inversiones en ciencia, innovación, generalización y la protección del medio ambiente.

\section{Sistema de indicadores para evaluar el impacto y la eficiencia de la gestión por} procesos, aplicación de 6 indicadores generales y 57 subindicadores o requisitos para medir el accionar de la entidad y programarlo en correspondencia con las prioridades de la estrategia territorial y el avance hacia la sostenibilidad.

¿Cómo se operacionaliza el modelo propuesto para la gestión integrada?

El proceso para la operacionalización del modelo propuesto, concibe 7 etapas y centra su realización en la segunda, tercera, cuarta y sexta etapa.

Etapa 1: Diagnóstico estratégico de la organización. Identificación de problemas.

Etapa 2: Planeación, organización, definición de política, prioridades y acciones estratégicas.

Etapa 3: Planificación de programas territoriales, planes tecnológicos, proyectos y asignación de financiamiento para ejecución de las acciones de la EPI. Elaboración del Plan trienal de ciencia, tecnología y sociedad (PTCITyS).

Etapa 4: Obtención de resultados, control y evaluación de impactos. Aplicación de indicadores integrales para evaluar la correspondencia de los resultados obtenidos con la satisfacción de las demandas identificadas en la sociedad y su vínculo con las prioridades estratégicas para el desarrollo económico social.

Etapa 5: Difusión de la información. Visibilidad e introducción de los resultados científicos y tecnológicos a través de la Red para la interfase.

Etapa 6: Evaluación de la eficiencia funcionamiento de la RED y el SCITy MA a través de los grupos de trabajo del Consejo Asesor Provincial. Sesiones de grupos de expertos y talleres en los organismos, municipios, empresas, entidades y organizaciones del territorio involucrados para evaluar anualmente la correspondencia de la oferta con la demanda tecnológica, a través de la introducción de 
los resultados de la ciencia en los destinos priorizados para el desarrollo territorial y su impacto en la sociedad.

Etapa 7: Seguimiento y control anual por los órganos reguladores de los resultados e impactos obtenidos. Aprobación de las acciones correctivas estratégicas, para su implementación y continuo perfeccionamiento en función del desarrollo sostenible y la calidad de vida de la sociedad.

Metodología estándar para la aplicación del modelo.

Diagnóstico.

Diseño de estrategia integrada.

Programa de gestión para los sistemas a implementar. (acciones, responsables, recursos y plazos de cumplimento).

Control y evaluación de impacto. (Por indicadores y subindicadores)

Aplicación de correcciones anuales para la mejora continúa.

\section{¿Qué se obtiene con la aplicación del modelo?}

Diagnóstico de innovación de la empresa o entidad.

Diseño de estrategia, políticas, programas y planes de acciones, en función de una gestión empresarial integral con base en el conocimiento y la innovación para el desarrollo.

Implantación paulatina de los sistemas estratégicos sobre políticas de innovación para el avance empresarial y social hacia el desarrollo sostenible.

Aplicación de indicadores para la evaluación de impacto de las acciones estratégicas planificadas.

Evaluación de los resultados de la empresa y su correspondencia con el entorno y el desarrollo de la estrategia territorial.

Obtención de Diploma con las categorías de: Empresa innovadora y Empresa innovadora por el desarrollo territorial sostenible. 


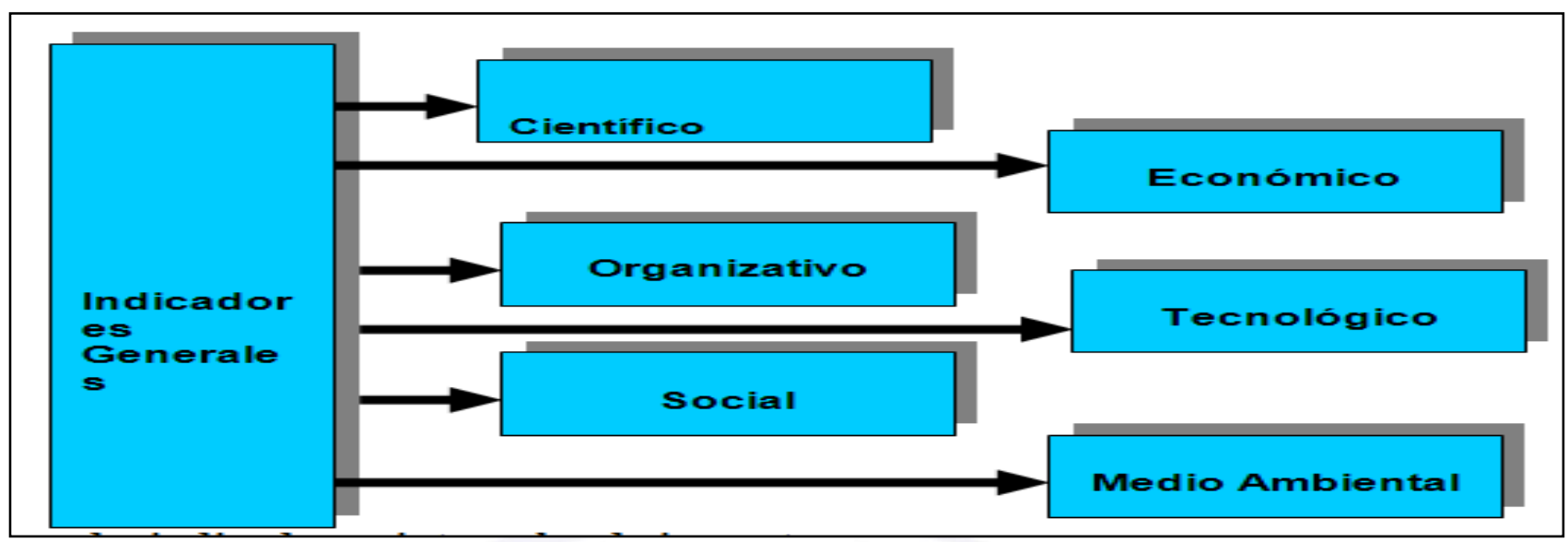

Figura 3 - Indicadores generales

Fuente: Nardo (2006)

\section{SISTEMA DE INDICADORES INTEGRALES DE IMPACTO}

Para la aplicación y evaluación de los indicadores de impacto propuestos, en todos los casos debe plantearse la medición en forma de incremento, decremento o asociación de algún factor de referencia respecto a un período de tiempo anterior, período este que consideramos deba ser como mínimo un año. Los valores deben ser expresados en términos de cantidades, de acuerdo a la unidad de medida que corresponde y en $\%$.

¿Cómo evaluar integralmente los impactos de la ciencia y la innovación tecnológica en la sociedad?

La medición y evaluación del impacto de la ciencia y la innovación tecnológica es un elemento importante a considerar en la evaluación del nivel de satisfacción de las necesidades prioritarias del desarrollo económico, social y productivo de la sociedad, por tanto el seguimiento y control del impacto de la ciencia debe ser también un elemento imprescindible para orientar la gestión y planificación de las acciones del SCIT y MA, en función de su contribución cada vez más decisiva al logro del objetivo estratégico del mejoramiento de la calidad de vida de los ciudadanos.

El sistema de indicadores integrales elaborados para evaluar el impacto de la ciencia y la innovación tecnológica, por su novedad obtuvo premio de la Academia de Ciencias de Cuba y aporta una herramienta para el perfeccionamiento de la gestión e implementación integrada del SCIT y MA, que utilizado de conjunto y a través del modelo alternativo propuesto (MOGECITECMA) contribuirá a materializar en la sociedad actual el nuevo enfoque hacia el desarrollo sostenible. 


\section{ANÁLISIS DE LOS RESULTADOS DE LA APLICACIÓN DE MOGECITECMA}

La aplicación del modelo resultante, visualizó tres importantes resultados:

- Mayor pertinencia de la correspondencia de los planes de las empresas y organizaciones con las prioridades estratégicas de la ciencia, la innovación y el medio ambiente y su contribución más puntual y dinámica con las demandas del desarrollo económico-social territorial y la elevación de la calidad de vida de la población.

- Procedimiento para la elaboración del plan trienal de ciencia, innovación y sociedad, conformación de programas, planes tecnológicos y proyectos científicos tecnológicos financiados territorialmente en correspondencia con las prioridades estratégicas para el desarrollo sostenible y las principales demandas de la sociedad.

- Identificación de los principales impactos de la ciencia, la innovación y el medio ambiente en la sociedad, como base para un mayor acercamiento de la demanda y la oferta tecnológica, para el continuo perfeccionamiento de la gestión de redes de ciencia e innovación.

La aplicación de los indicadores elaborados para la medición del impacto de la ciencia y la tecnología en la sociedad permite evaluar el comportamiento de los resultados obtenidos aplicar el modelo y reorientar las políticas de innovación, los planes estratégicos de las empresa y organizaciones hacia las demandas básicas y prioridades identificadas para el desarrollo sostenible del territorio.

\section{CONCLUSIONES}

1. Los avances de la ciencia contemporánea y el uso acelerado de nuevas tecnologías, con sus respectivos impactos económicos y sociales demandan cada vez cambios radicales, en las políticas de los sistemas de innovación, modelos organizativos y paradigmas para la gestión de la ciencia y la innovación tecnológica. Solo los enfoques integrales y multidisciplinarios sobre el tema, acompañados de la dimensión social, ambiental y cultural orientarán a las presentes y futuras organizaciones hacia una política de innovación dirigida al desarrollo sostenible y el logro de los objetivos propuestos. 
2. MOGECITECMA, nuevo modelo diseñado, para la gestión de la ciencia y la innovación tecnológica constituye una innovación organizacional en los procesos de perfeccionamiento institucional que se realizan en Cuba ha contribuido a lograr que Villa Clara, como caso de estudio elabore y gestione una política de innovación en función del desarrollo sostenible y logre impactos sostenidos en la sociedad y sea considerada de referente nacional.

3. Los indicadores de impactos elaborados en el modelo MOGECITECMA están aprobados y se aplican actualmente en proyectos territoriales, nacionales e internacionales.

\section{REFERENCIAS}

Echeverria, J. (1995). Filosofía de la ciencia. Madrid. Akal.

González Garcia, M., Lopez Cerezo, J. A., \& Luján, J. L. (1996). Ciencia, tecnología y sociedad: una introducción al estudio social de la ciencia y la tecnología. Madrid: Tecnos.

Nardo, M. A. S. (2006). Un modelo para la gerencia integrada de la ciencia, la innovacion tecnologica y el medio ambiente: estudio de casos en Villa Clara (p. 4). Memórias de Taller Internacional de Gestión Tecnológica, 10. Cuba: Gestec. 


\title{
A NEW APPROACH TO THE MANAGEMENT OF INNOVATION POLICIES IN THE ROLE OF SUSTAINABLE DEVELOPMENT
}

\begin{abstract}
The globalization wave that characterizes the XXI century is distinct from former waves because of the speed of technological changes and its impact on policies and organizational models for management of technology. The drive of events brings, in pushing ways, issues related to science, such as its appropriate use in the society, the militarization of science, the relationship of science with government, scientific secrets, the freedom of science, the role and place of science in the education and in the general culture. How to solve such problems? So the challenge under the perspective of science integration, innovation and society call for new multidisciplinary research to deepen theoretically and practically, how to get effective systems of innovation more competitive in a more human society. Considering the challenge of a new vision for the XXI century, we conceptualize, sketch and participate in the direction of an innovative experience on knowledge management that embodies a new focus of socio-philosophical debate issues on the construction of new paradigms for the management of innovation for current days. The scientific value of this work resides on the novelty of socio-philosophical studies in Cuba, on this particular theme. The author brings a new focus for innovation policies highly integrated with the environment. The proposed concept originates in the case study of Villa Rica Provence, in Cuba, resulting in an innovation system, called MOGECITECMA, more competitive and integrated because it opens up the opportunity for advancements for a sustainable community.
\end{abstract}

Keywords: Innovation; Innovation systems; Sustainable innovation.

Data do recebimento do artigo: 25/05/2010

Data do aceite de publicação: 04/08/2010 